\title{
EL PORTAFOLIO ETNOGRÁFICO: UNA HERRAMIENTA FACILITADORA DEL APRENDIZAJE A LO LARGO DE LA VIDA
}

\author{
THE ETNOGRAPHIC-PORTFOLIO: \\ A TOOL TO FACILITATE $A$ LONGLIFE LEARNING
}

\author{
Ana Arraiz Pérez* y Fernando Sabirón Sierra**
}

Facultad de Educación de la Universidad de Zaragoza

\begin{abstract}
RESUMEN
Consecuencia de un ensayo en innovación educativa, contrastado en la propia experiencia docente, presentamos nuestra concepción del portafolio etnográfico. Se trata de una herramienta que ayuda en el proceso personal de formación continuada para el ejercicio de la ciudadanía, como una forma de superar el dilema existencial entre los intereses sistémicos y el desarrollo autodeterminado de los individuos. Se analiza desde su alto potencial mediador para comprometer un aprendizaje constructivista, en términos de evaluación de logros y reorientación de proyectos. Genera dossieres significativos con y para los Otros, con la entrevista como elemento clave.
\end{abstract}

Palabras clave: Portafolio. Aprendizaje a lo largo de la vida. Intersubjetividad. Complejidad. Entrevista.

\begin{abstract}
As a consequence of an essay in educational innovation, fried out in our own educational experience, we outline our conceptualization of ethnographic-portfolio. It is a the process of personal tool which helps in the process of personal development to exert citizenship, as a way to over come the existential dilemma between systemic interests and the person's self-determined development. Its high mediating potential is discussed to engage in a constructivist learning process, in terms of an achieve-

* Dra. Ana Arraiz Pérez, Profesora Titular de Universidad, área de Métodos de Investigación y Diagnóstico en Educación. Investigadora principal del Grupo de Investigación Aplicada en Etnografía de la Educación (EtnoEdu), desarrolla la línea sobre orientación educativa y déviance. Entre sus últimas publicaciones, reseñar $L a$ complejidad narrativa de la orientación y el diagnóstico en Educación (Zaragoza: Mira Editorial). [aarraiz@ unizar.es]

** Dtor. Fernando Sabirón Sierra, Profesor Titular de Universidad, área de Métodos de Investigación y Diagnóstico en Educación. Director del Grupo de Investigación Aplicada en Etnografía de la Educación (EtnoEdu), desarrolla la línea sobre educación no-formal y déviance. Entre sus últimas publicaciones, reseñar Métodos de Investigación Etnográfica en Ciencias Sociales (Zaragoza: Mira Editorial). [fsabiron@unizar.es]
\end{abstract}


ment evaluation and a revision of projects. It generates significant dossiers with and for the Others. The interview is the essential component.

Key words: Portfolio. Longlife learning. Intersubjectivity. Complexity. Interview.

\section{Contextualización introductoria}

Desde que existimos, intrínseca a nuestra naturaleza humana está la Educación; en las últimas décadas hemos querido mantener al alza un sueño histórico, el de cómo en ella, actualizada ahora entre la denominación del aprendizaje a lo largo de la vida (OCDE, 2004) y la persistencia en la crítica de una compleja intersección planetaria de la existencia (Morin, Ciurana y Motta, 2003), no solo consigue la persona su emancipación, sino que incluso la Humanidad en su conjunto se ve beneficiada. Sin embargo, un fantasma recurrente vaga en el sueño: cómo resultando tan sencillas en apariencia las acciones educativas, no parece fácil lograr estrategias y técnicas mediadoras en una Educación referenciada desde esa utopía; incluso al asesoramiento, cuya específica función incide precisamente en promover el cambio y mejora de cualesquiera de las situaciones de la vida, le resulta complicado potenciar el progreso hacia la auto-orientación de cada persona. Así es como se comprende la tendencia compulsiva a adoptar sin reservas cualquier herramienta en la que se intuya potencial para desarrollar nuestras polivalentes intenciones, sin importar demasiado sus referentes epistemológicos: el portafolio es un ejemplo paradigmático. Las prácticas evaluativas resultan ser uno de los indicadores diagnósticos más relevantes en el análisis de las acciones educativas en general y orientadoras en particular. La evaluación, sin embargo, tiene añadida una característica que la hace particular: una simplicidad de instrumentos, técnicas y estrategias que contrasta profundamente con la complejidad de la función. El portafolio, del francés portefeuille, "cartera de mano para llevar libros, papeles, etc.", definición que nos brinda la Real Academia Española, puede ser perfectamente un digno heredero de la simplicidad instrumental en la complejidad efectiva de la praxis evaluativa. Así lo mantiene Shulman $(1999,45)$ cuando se empeña en demostrar que el portafolio es un acto teórico. El portafolio es capaz de convulsionar la formación, porque demanda y nos exige un cuestionamiento de nuestra praxis; a la vez que puede ser capaz de facilitar el proyecto personal y profesional, potenciando mayor satisfacción y calidad de vida al provocar la narración reflexiva de la existencia. Es cierto, el portafolio está de moda, pero ¿qué sentido, significado y uso del portafolio garantizan que pueda superar su condición de complemento de temporada para transformarse en elemento de fondo de armario? La respuesta implica un doble proceso deconstructivo-reconstructivo: desde la indagación documental en significados atribuidos, el análisis de los referentes que le dan sentido, para concluir con la acotación del portafolio etnográfico, como propuesta comprometida con la complejidad de un aprendizaje a lo largo de la vida, renovador del sentido de la persona en el mundo.

\section{La ambigüedad conceptual del portafolio.}

A diferencia de otros dominios, existe consenso técnico en la determinación de distintos tipos de portafolio asociados al uso que se le atribuya: es portafolio de presentación el que realza los logros máximos en términos de productos, y será portafolio de evaluación, aquel 
utilizado con este fin (y así sucesivamente). El sentido comercial, introdujo en origen una característica que se mantiene: el logro depende tanto de la propia producción, como del ajuste al juicio del comprador. La capacidad y externalidad de la venta, la acreditación, la calidad dícese, constituyen rasgos distintivos de la motivación histórica reciente por la que se introduce, en las alternativas de evaluación, el portafolio. Con inusitada rapidez se incorpora el instrumento en distintos sistemas educativos: EE.UU. y Canadá, pero principalmente el segundo enfatiza el portafolio como herramienta de aprendizaje ${ }^{1}$. Es así como aparecen las distintas modalidades de aplicación referidas tanto al producto como al proceso, unidas a las iniciales del portafolio profesional, adaptándose, a su vez, en el próximo y anticipador desarrollo para la carrera (Ibarra y Sánchez, 1999). La reorientación del saber escolar, de los sistemas formativos, como consecuencia de la evolución socio-productiva, recupera el interés desmedido por intervenir sobre el recurso humano desde la formación continuada. El portafolio es, desde esta perspectiva, uno de los instrumentos que propicia la renovación por una recuperación del liderazgo y la competitividad laboral (Stewart, 2003). La competencia es el artilugio tecnicista que representa esta inclinación de intervención sobre los recursos humanos; el portafolio, le añade un plus de calidad digno de encomio: ahora al saber hacer acreditado por un agente externo, le sumo el sentirme satisfecho por ser consciente del cómo lo he adquirido, y orgulloso cuando evidencio el dominio de mi saber hacer en sus mejores muestras; hasta tal punto, que incluso el dominio del propio instrumento es esencial para moverse en el dinámico e impredecible mercado de trabajo. Y de su importancia, a la complejidad de su elaboración, tal y como puede inferirse de la guía canadiense de estándares y competencias del orientador profesional (Canadian Standards and Guidelines for Career Development Practitioners, 2004) que selecciona de manera explícita la orientación al cliente en el uso del portafolio. El origen del instrumento, por su forma y sentido marcadamente pragmático, encuentra pronto acomodo en el formato virtual. El portafolio electrónico domina tanto los contenidos de las páginas de empresas que quieren presentar sus productos, como las webs dedicadas a las proliferantes innovaciones didácticas (Vanhuelle y Schillings, 2003). Cuando Ashman (2004) metaevalúa el material disponible en red, configura un perfil exhaustivo de la diversidad actual del portafolio como instrumento de aprendizaje y evaluación, en particular, la de documentar la trayectoria profesional. La disparidad de uso es manifiesta, pero cabe reforzar una evaluación en positivo porque se pide aquello que se puede alcanzar, y no lo que la persona no puede hacer. Si bien, hay que añadirle la variedad de riesgos, debilidades y fortalezas para aproximarnos al retrato impresionista del portafolio. Shulman $(1999,59-62)$ apunta los abusos por exceso de exhibición, trivialización, o representación errónea en que puede incurrir el portafolio; pero a la vez, avala la consideración del portafolio en tanto que refuerza el seguimiento, la reconexión proceso-producto, o la reflexión y el análisis, favoreciendo el desarrollo de macro-competencias profesionales básicas. El valor más importante del portafolio es, sin embargo, cambiar el centro de la actividad que pasa a manos del practicante; redefiniendo la actividad, sea ésta docente, orientadora, evaluativa o investigadora. Es este retorno humanista hacia el protagonismo de la persona — "los portafolios (también) traen gente dentro", en síntesis de Sá-Chaves (2005) — la clave de éxito en nuestro sentido de portafolio.

1. Canadá difiere sustancialmente de EE.UU. El uso canadiense refleja una preocupación por los desajustes en las prácticas evaluativas (Scallon, v. gr.). En nuestra concepción del portafolio nos adscribimos a esta línea. 


\section{El uso del portafolio: una deconstrucción interesada de referentes claves}

A la vista de la situación esbozada, nuestra presentación dialéctica del portafolio implica la consideración de una perspectiva éticamente correcta en cuanto puede servir a una función ilustradora de la persona, y otra incorrecta desde la que sirve a los intereses de reproducción, en un sistema por naturaleza desigual y no siempre justo. La fundamentación nos remite, a su vez, a tres referentes vertebradores de su recreación en el aprendizaje a lo largo de la vida: las competencias, en su sentido práctico; la evaluación de competencias, en tanto que función sistémica; y el constructivimo social, como sentido teórico del desarrollo dialéctico de la persona en su mundo.

\section{Competencia}

Resulta banal señalar que no existe en nuestro campo — una vez más — acotación conceptual unívoca: no hay un marco teórico único que recoja el uso instituido del constructo competencia (Stoof et al., 2002; Roegiers 2004). Por ello, cabe destacar algunas investigaciones realizadas en nuestro contexto y referidas al orientador tanto bajo una perspectiva europea (Repetto, Ballesteros y Malik, 1999), como en la dimensión localista (García-Fuentes, Rodríguez Rodríguez y Rodríguez Fernández, 2006) que nos ofrecen el desglose competencial con una directa vinculación tanto a la formación como al propio desarrollo de la profesión. De la síntesis que realiza Castellano (2005), en este caso relativa a las competencias culturales, es de interés igualmente la asociación entre competencia y actividad, a la vez que vincula la persona, con grupos y organizaciones. No obstante, el término competencia sí remite, en principio, a dos referentes distintos tanto como constructo teórico, cuanto como expresión de una valoración (Louis, Jutras y Hensler, 1996). En el sentido de enjuiciamiento y valoración, competencia es asimilable a estándar de desempeño; en la concepción profesionalizada se asocia a situaciones de trabajo ... pero: ¿quién define la situación? En nuestro caso, la multirreferencialidad, la complejidad y el caos, interaccionismo y socio-constructivismo, ofrecen una alternativa clarificadora acorde con la prospectiva del mercado laboral, hilo conductor del aprendizaje a lo largo de la vida. La definición de Meirieu (2004), como saberes relativos a situaciones complejas que implican dominar variables heterogéneas para resolver problemas que escapan a situaciones referidas epistemológicamente a una sola disciplina, resulta primordial en tanto que referente básico que regula nuestra concepción.

\section{Evaluación}

El portafolio, en tanto que instrumento en procesos formativos competenciales, así como estrategia de desarrollo personal que favorece el pensamiento crítico, puede resultar significativo en el afrontamiento de la persona respecto a las cortapisas sistémicas que condicionan las prácticas evaluativas. Sin embargo, es desde la evaluación donde centramos ahora el potencial alternativo, atendiendo a la evolución terminológico-conceptual que, en la línea de la evaluación formativa, alcanza la denominada evaluación auténtica. Wiggins (1989) pasa por ser uno de los primeros que utilizó el calificativo de "auténtico" aplicado a 
los tests; en concreciones posteriores, Wiggins (1990) precisa condiciones de la aludida autenticidad en torno a dos criterios: "refleja desafíos, trabajo y estándares vigentes en el ejercicio profesional"; a la vez que, "compromete verdaderamente al alumno en forma interactiva con oportunidades para explicar, dialogar e investigar". Evaluación auténtica y portafolio se irán reforzando mutuamente. En todo caso, el portafolio se propone como instrumento propio de una genéricamente considerada evaluación alternativa, tanto por la contextualización de los saberes (evaluación auténtica), como por el refuerzo de la función de acompañamiento y mejora (evaluación formativa). Si bien, es preciso resaltar una vez más la capacidad vehicular respecto a la relación educativa; dado que, tal y como refleja Scallon (2003) el portafolio permite incorporar una lógica evaluativa distinta derivada del compromiso de la persona en su calidad de aprendiz con los agentes evaluadores. Únicamente desde la colaboración cobra el sentido auténtico la evaluación por portafolio etnográfico.

\section{Socio-constructivismo}

Dos son los elementos conceptuales particularmente destacables como bases para fundamentar la propia redefinición etnográfica del portafolio: la zona de desarrollo potencial y la indagación dialógica. Aprender con los demás y de los demás, en la interacción implicada del actuar, pensar y sentir de la persona, en una construcción compartida de la propia acción, desde la intersubjetividad y alteridad que transforma, en definitiva, a los individuos y a las organizaciones: todo ello va a mediar el proceso interno y externo de elaboración del portafolio. Desde nuestra concepción del socio-constructivismo, es además particularmente relevante el portafolio por cuanto facilita — entre otros referentes - la aplicación de técnicas narrativas. Conviene referir la posición de Lyons (1999, 143-163) en la vinculación particular de la entrevista y el portafolio, señala: "La entrevista sobre el portafolio bien puede hacer las veces de ese andamiaje requerido. Primero el portafolio se construye y se presenta (...) luego, en una segunda entrevista, se reflexiona sobre la producción del portafolio, y se especifica el sentido que se atribuye a las experiencias". La entrevista resulta, en nuestra propuesta, no menos decisiva.

\section{El portafolio etnográfico: la reconstrucción de una herramienta orientadora}

Las pinceladas precedentes permiten acotar el significado de portafolio adoptado, que cobra sentido en la orientación para el ejercicio de la ciudadanía. Se trata de una herramienta mediadora en el aprendizaje constructivista, que implica la elaboración de dossieres significativos e intersubjetivos — con y para los otros_- pero siempre desde el sentido que le confiere cada individuo, como persona en permanente crecimiento comprometido con su entorno, en definitiva son vivencias narradas en proyección de futuro. El calificativo etnográfico devuelve y apuntala tal mediación del portafolio, al menos desde dos aspectos de particular relevancia:

- El desvelamiento y crítica emergente a los procesos de conocimiento en los que el individuo se compromete a lo largo de su ciclo vital y que, desde su protagonismo en el proceso, transforma en acción; uno recoge en su portafolio su singularidad, pero apor- 
ta al colectivo saberes; siendo intransferible, la documentación es, sin embargo, socializable. Se enlaza aquí con la autopista de las competencias profesionales, resultantes de la asignación de una capacidad de acción profesional fundamentada en el conocimiento, cuyo referente es la praxis. Recurre a la complejidad, al considerar el constructo en tanto que conjunto de fenómenos complejos en interacción (Sabirón, 2006). Apela a la multirreferencialidad explicativa, comprensiva, aplicativa y evaluativa, dadas las categorías heterogéneas y emergentes a cada caso. Narra el proceso en un lenguaje interdisciplinar presto a la referencia transdisciplinar, a la vez que adquiere el significado — finalmente - en el compromiso con la persona. Desde este perfil, la evaluación entroncada en el portafolio etnográfico contempla la regulación sistémica (acreditación incluida) como punto de arranque, desde la contemplación reflexiva, a la reconstrucción personal en el desarrollo de la auténtica autodeterminación. El "empowerment" implícito cuestiona incluso las posibilidades que los entornos ofrecen a los individuos para su aprendizaje competencial.

- El acompañamiento preciso para orientar un proceso de conocimiento constructor de realidades, evitando la trivialización de la herramienta, o falsos espejismos de soliloquios individuales. El instrumento precisa de asesoramiento, son necesarios los Otros, lo colectivo; porque ante todo y sobre todo, el portafolio etnográfico es una herramienta intersubjetiva. Como consecuencia natural, es inseparable de la entrevista que articula y en la que se recrea; condicionada a su vez por el mediador que la lidera. Quien si no propicia procesos de reflexión crítica en el conflicto intersubjetivo, difícilmente permitirá que el portafolio supere su acepción de carpeta de trabajo. Así pues, el profesional del acompañamiento — orientador-, no puede limitarse a transmitir significados al uso del portafolio respecto al proyecto personal y profesional del destinatario de la acción orientadora; ni prescribir únicamente un formato con potencial suficiente para manejarse en el mundo del trabajo. Por el contrario, debe comprometerse con el usuario en un proceso de indagación dialógica que, venciendo resistencias, propicie superar la consabida oposición dilemática entre sistema y mundo de vida, hacia un nivel de realidad que transforma persona y entorno: la utopía educativa más allá de la globalización.

Pero, ¿cómo formatear un portafolio etnográfico para que, conservando la simplicidad del instrumento incorpore la complejidad de tal función teórico-práctica? Resulta cuando menos obvio afirmar que sólo en el contexto de la comunicación puede lograrse un formato cuasi-definitivo de portafolio, que se irá reificando a lo largo del proceso por su dinamismo intrínseco; pero no es menos cierto que se impone dar forma y estructura inicial a la herramienta, como tema de fondo de la entrevista inicial y de cuántas resulte pertinente establecer. Carpetas y criterios de evaluación son así los ejes vertebradores del formato del portafolio. La selección de las carpetas es uno de los asuntos más polémicos en la configuración de la herramienta y que suscita un debate más comprometido. Su denominación va a condicionar, a modo de organizador previo, clase y categoría de artefactos y procesos a integrar, influyendo necesariamente en los aprendizajes a conseguir. De tal forma, que si las carpetas propuestas versan sobre competencias muy estrechamente vinculadas a circunstancias momentáneas bien sean laborales, escolares, familiares o personales, el desarrollo competencial promovido será a corto o medio plazo; mientras que si por el contrario, se proponen competencias generales, transversales a distintas profesiones y proyectos de vida, abordando previsiones y posibilidades, la formación incorporará la proyección de futuro imprevisto 
pero posible, estrechamente unida al aprendizaje permanente; máxime, cuando este tipo de carpetas incide preferentemente en fortalezas por lo producido y lo proyectado, con independencia del tipo de lenguaje que se utilice, a menudo mediatizado por el pretexto que justifica el inicio de la herramienta ${ }^{2}$. Por otra parte, si se acota de manera unívoca la denominación de las carpetas, se especifican de manera exhaustiva los productos y procesos a documentar, o se enfatiza el desarrollo de componentes técnicos por encima de los cognitivos, actitudinales y axiológicos, disminuirá notablemente el proceso colaborativo, y lo que es más grave, se limitará sustancialmente la autorreflexión y autoevaluación, funciones determinantes en el portafolio etnográfico. Precisamente, los criterios de evaluación seleccionados servirán, en particular, para consolidar el formato iniciado con la selección de las carpetas y, en general, para avanzar hacia la consolidación de un aprendizaje complejo, referente primero de la evaluación etnográfica.

\section{Referencias bibliográficas}

Arraiz, A. y Sabirón, F. (2005). "El portafolio, una herramienta intersubjetiva para la evaluación etnográfica de competencias". En Actas AIOSP/IAEVG Internacional Conference, Lisboa, 14-16 de septiembre.

Ashman, J. (2004). Le portfolio, un outil de communication et d'évaluation des compétences? Bruxelles: Direction de la Formation de la Police Belge.

Campbell, P. H. (2001). "Strengths-based child portfolios". Topics in Early Childhood Special Education, 21 (3), 152-161.

Castellano, J. L. (2005). "Competencias interculturales en servicios de orientación para el desarrollo de la carrera". Revista Española de Orientación y Psicopedagogía, 16 (1), 13-29.

García-Fuentes, C. D.; Rodríguez, O. y Rodríguez, S. (2006). "Características competenciales del psicopedagogo: una aproximación desde la Comunidad Autónoma de Galicia". Revista Española de Orientación y Psicopedagogía, 17 (1), 91-106.

Ibarra, M. y Sánchez, C. (1999). "Posibilidades del portafolios en auto-orientación profesional". Revista Española de Orientación y Psicopedagogía, 10 (7), 63-82.

Louis, R.; Jutras, F. y Hensler, H. (1996). « Des objectifs aux compétences ». Revue Canadienne de l'Éducation, 21 (4), 414-432.

Lyons, N. (1999). El uso de portafolios. Buenos Aires: Amorrortu editores.

Meirieu, Ph. (2004). L'Envers du tableau. Quelle pédagogie pour quelle école? Paris: ESF.

Morin, E., Ciurana, E. R. y Motta, R. (2003). Educar en la era planetaria. Barcelona: Gedisa.

National Steering Committee for Career Development Guidelines and Standards (2004). Canadian Standards and Guidelines for Career Development Practitioners. Ottawa (ON): HRDC. Consultado en febrero de 2005 en http://www.career-dev-guidelines.org/.

OCDE (2004). Orientación profesional y políticas públicas. Paris: OCDE-MEC.

Repetto, E., Ballesteros, B. y Malik, B. (1999). "Hacia una formación de los orientadores en Europa: estudio empírico de las áreas de competencias más relevantes". Revista Española de Orientación y Psicopedagogía, 10 (17), 149-162.

2. A modo de ejemplo, referir formatos aparentemente distintos, con el común denominador de incrementar las posibilidades del proyecto personal y profesional: Campbell (2001), Stewart (2003), y Arraiz y Sabirón (2005). Para ampliar nuestra posición, puede consultarse http://www.unizar.es/etnoedu. 
Roegiers, X. (2004). Compétence, compétence ou compétence ? Consultado en febrero de 2005 en http://www.bief.be /enseignement/ publication/Terminologie_competences.pdf.

Sabirón, F. (2006). Métodos de investigación etnográfica en Ciencias Sociales. Zaragoza: Mira editores.

Sá-Chaves, I. (2005). Os "portfolios" reflexivos (tambén) trazem gente dentro. Porto: Porto editora.

Scallon, G. (2003). L'harmonisation des fonctions formative et sommative de l'évaluation. Québec: Presses de l'Université de Laval.

Shulman, L. (1999). "Portafolios del docente: una actividad teórica". En N. Lyons (ed.), El uso de portafolios (pp. 44-62). Buenos Aires: Amorrortu editores.

Stewart, J.B. (2003). "Using Portafolios to Direct Workplace Learning”. The Canadian Journal of Career Development, 21 (1), 26-32.

Stoof, A. et al. (2002). "The boundary approach of competence". Human Resource Development Review, 1 (3), 1-32.

Vanhuelle, S. y Schillings, A. (2003). Avec le portfolio. Bruxelles: Labor.

Wiggins, G. (1989). “A True Test: Toward More Authentic and Equitable Assessment”. Phi Delta Kappan, 70 (9), 703-713.

Wiggins, G. (1990). "The case for authentic assessment". Practical Assessment, Research \& Evaluation, 2(2). Consultado en febrero 2003 en http://PAREonline.net/getvn.asp?v=2\&n=2.

Fecha de recepción: 10-01-06

Fecha de revisión: 20-04-07

Fecha de aceptación: 08-05-07 